\title{
The 2013 special issue on stem cell biology
}

\author{
Cell Research (2013) 23:1-2. doi:10.1038/cr.2013.4; published online 3 January 2013
}

Stem cells hold the remarkable capacity of self-renewal and differentiation into more specialized cell lineages, and thus constitute a promising resource in regenerative medicine for the generation of appropriate cell types in cell replacement therapy. Stem cell research, accordingly, has become a highly vigorous and rapidly evolving field in life sciences, highlighted by the 2012 Nobel Prize in Physiology or Medicine awarded to Shinya Yamanaka and John Gurdon, for their ground-breaking works in reprogramming cell fates [1]. As a general interest life science journal, Cell Research has enjoyed a phase of rapid growth in the past several years, as shown by the dramatic improvement in the scientific quality of papers published in the journal as wells as its broadening impact within the scientific community. Along the way, the field of stem cell biology has naturally become an important research area covered by papers published in Cell Research [2]. For instance, in the past year of 2012, Cell Research has published a number of important papers related to the stem cell field, covering diverse aspects and topics such as induced pluripotent stem (iPS) cells [3-7], mechanistic studies of pluripotency and differentiation [8$11]$, modeling of human diseases using stem cell-based systems [6, 12], direct reprogramming of somatic cells to other cell types without passing through an pluripotent intermediate [13-16], as well as neural crest stem cells [17] and cancer stem cells [18-20].

Reflecting our continued interest and building on our existing strength in this area, we are excited to present to our readers this 2013 Cell Research Special Issue on "Stem cell biology". This spe- cial issue consists of two major parts. The first part of the issue presents a collection of 5 review articles authored by world-renowned scientists in the respective fields. Two reviews focus on the basic regulatory mechanisms in ES and iPS cells: Huck-Hui Ng and colleague review our current understanding of the transcriptional regulatory principles underlying the state of pluripotency in ES cells and discuss how the control of various signaling pathways could influence pluripotency; the article by Yi Zhang and colleague presents a comprehensive overview of both the epigenetic landscapes of pluripotent stem cells and epigenetic mechanisms involved in iPS cell generation. The article by George Daley and colleague provides us a state-of-art analysis of the current understanding on cell fate and cell fate conversions, covering diverse aspects such as iPS cells and their differentiation, direct conversion of cell fates between differentiated states, as well as facilitated lineage switching through partial reprogramming. Much of the excitement of the iPS cell technology stemmed from its potential use in regenerative medicine, and it is clear that significant research efforts are still needed before this potential could be realized. In their article, Hideyuki Okano and colleague review the progress from pre-clinical studies that attempted to treat spinal cord injury using neural stem/progenitor cells derived from iPS cells, and discuss strategies and safety issues related to iPS cell-based therapies. Finally, Sheng Ding and colleagues discuss chemical approaches in the study of stem cell biology, which have proved to be a powerful tool for improving our understanding of the basic biology of stem cells as well as for engineering cell fate changes toward desired cell types.

The second half of the special issue comprises 5 original research articles, among which three are related to iPS cells. Improving the quality of iPS cells will be critical for their potential applications in cell-based therapy. In their research article, Jinsong Li and colleagues report that including a factor called Zscan 4 in the reprogramming cocktail could greatly improve the quality of the resulting iPS cells, as demonstrated by the tetraploid complementation assay, and suggest that this factor likely acts to promote genomic stability during the reprogramming process. In two other articles, Xin Xie and colleagues report that a simple environmental stress, hyperosmosis, could promote reprogramming via p38 activation, while Jiuhong Kang and colleagues show that a microRNA called miR-29b, acts to mediate the function of Sox 2 in reprogramming, and that it does so via regulating DNA methyltransferases. Mesenchymal stem cells (MSCs) have been shown to possess immunomodulatory properties. The paper by Songtao Shi and colleagues reports the rather surprising finding of a subset of MSCs that express IL17, fail to execute immunomodulation, and exhibit an inhibitory effect against an infectious fungus. Finally, the research article by Yi Zhang and colleagues reveals novel roles of $\mathrm{H} 3 \mathrm{~K} 27 \mathrm{me} 3$ demethylases KDM6A and KDM6B in endoderm differentiation from human ES cells via regulation of Wnt signaling. The issue also includes two brief letters to the editor, which report new advances in generating functionally more mature hepatocytes from human ES cells, and 
in the generation of cloned animals from pig iPS cells, respectively.

We would like to thank our Guest Editors, Linzhao Cheng, Hongkui Deng, Linheng Li, and Haifan Lin for their help in the commissioning of the review articles, and all of the authors for their contributions to this special issue. We hope this exciting collection of articles on stem cell biology will be an enjoyable read and will contribute to the growth of the field in years to come.

\section{Dangsheng $\mathrm{Li}^{1}$}

${ }^{1}$ Deputy Editor-in-Chief, Cell Research, Shanghai Institutes for Biological Sciences, Chinese Academy of Sciences, Shanghai 200031, China

dsli@sibs.ac.cn

\section{References}

1 Abbott A. Cell rewind wins medicine Nobel. Nature 2012; 490:151-152.

2 Li D. A special issue on cell signaling, disease, and stem cells. Cell Res 2012; 22:1-2.

$3 \mathrm{Xu} \mathrm{H}$, Yi BA, Wu H, et al. Highly efficient derivation of ventricular cardiomyocytes from induced pluripotent stem cells with a distinct epigenetic signature. Cell Res 2012; 22:142-154.

4 Panopoulos AD, Yanes O, Ruiz S, et al. The metabolome of induced pluripotent stem cells reveals metabolic changes oc- curring in somatic cell reprogramming. Cell Res 2012; 22:168-177.

5 Cao N, Liu Z, Chen Z, et al. Ascorbic acid enhances the cardiac differentiation of induced pluripotent stem cells through promoting the proliferation of cardiac progenitor cells. Cell Res 2012; 22:219-236.

6 Wang $\mathrm{Y}$, Zheng CG, Jiang Y, et al. Genetic correction of $\beta$-thalassemia patient-specific iPS cells and its use in improving hemoglobin production in irradiated SCID mice. Cell Res 2012; 22:637-648.

7 Wang F, Yin Y, Ye X, et al. Molecular insights into the heterogeneity of telomere reprogramming in induced pluripotent stem cells. Cell Res 2012; 22:757-768.

8 Ding J, Xu H, Faiola F, Ma'ayan A, Wang J. Oct4 links multiple epigenetic pathways to the pluripotency network. Cell Res 2012; 22:155-167.

9 Patterson M, Chan DN, Ha I, et al. Defining the nature of human pluripotent stem cell progeny. Cell Res 2012; 22:178-193.

10 Wang C, Tang X, Sun X, et al. TGF $\beta$ inhibition enhances the generation of hematopoietic progenitors from human ES cell-derived hemogenic endothelial cells using a stepwise strategy. Cell Res 2012; 22:194-207.

11 Gwak J, Hwang SG, Park HS, et al. Small molecule-based disruption of the Axin/ $\beta$-catenin protein complex regulates mesenchymal stem cell differentiation. Cell Res 2012; 22:237-247.

12 Bueno C, Montes R, Melen GJ, et al. A human ESC model for MLL-AF4 leu- kemic fusion gene reveals an impaired early hematopoietic-endothelial specification. Cell Res 2012; 22:986-1002.

13 Sheng C, Zheng Q, Wu J, et al. Direct reprogramming of Sertoli cells into multipotent neural stem cells by defined factors. Cell Res 2012; 22:208-218.

14 Liu X, Li F, Stubblefield EA, et al. Direct reprogramming of human fibroblasts into dopaminergic neuron-like cells. Cell Res 2012; 22:321-332.

15 Meng F, Chen S, Miao Q, et al. Induction of fibroblasts to neurons through adenoviral gene delivery. Cell Res 2012; 22:436-440.

16 Sheng C, Zheng Q, Wu J, et al. Generation of dopaminergic neurons directly from mouse fibroblasts and fibroblastderived neural progenitors. Cell Res 2012; 22:769-772.

17 Achilleos A, Trainor PA. Neural crest stem cells: discovery, properties and potential for therapy. Cell Res 2012; 22:288-304.

18 Tang DG. Understanding cancer stem cell heterogeneity and plasticity. Cell Res 2012; 22:457-472.

19 Chen T, Yang K, Yu J, et al. Identification and expansion of cancer stem cells in tumor tissues and peripheral blood derived from gastric adenocarcinoma patients. Cell Res 2012; 22:248-258.

20 Hou Y, Zou Q, Ge R, Shen F, Wang Y. The critical role of $\mathrm{CD} 133^{+} \mathrm{CD} 44^{+/ \text {high }}$ tumor cells in hematogenous metastasis of liver cancers. Cell Res 2012; 22:259272. 